\title{
AVALIAÇÃO DA FUNÇÃO SEXUAL DE MULHERES NA MENOPAUSA
}

Jéssica Mariany Rodrigues da Silva ${ }^{1}$, Dárida Pereira Valeriano ${ }^{1}$, Guilherme Yassuyuki Tacao ${ }^{2}$, Lara Ribeiro Pereira $^{1}$, Leticia Pereira Santos ${ }^{1}$, Mayane Santos Arantes ${ }^{3}$, Raiza Mayara Gutierres Magalhaes ${ }^{4}$, Thaisa Paula Félix da Silva ${ }^{3}$, Franciele Marques Vanderlei ${ }^{1}$, Edna Maria do Carmo ${ }^{1}$

${ }^{1}$ Universidade Estadual Paulista - UNESP, Presidente Prudente, SP. ${ }^{2}$ Universidade Estadual do Norte do Paraná UENP. ${ }^{3}$ Universidade do Oeste Paulista - UNOESTE, Presidente Prudente, SP. ${ }^{4}$ Universidade do Sagrado Coração - USC, Bauru, SP.e-mail; jemariany@hotmail.com

\section{RESUMO}

O climatério pode apresentar alterações hormonais, fisiológicas, metabólicas e psicossociais, que repercutem na vida e no interesse sexual. 0 objetivo foi avaliar e classificar a função sexual de mulheres na menopausa. Foram recrutadas 84 mulheres para a pesquisa, das quais 57 se enquadraram nos critérios de inclusão: encontrar-se na menopausa (cessação da menstruação por, no mínimo, 12 meses) e ter tido vida sexual ativa nos últimos seis meses. Do Quociente Sexual versão feminina foram avaliados os domínios: desejo e interesse sexual, preliminares, excitação pessoal e sintonia com o parceiro, conforto, orgasmo e satisfação. O escore total do QS-F determinava o padrão de desempenho/satisfação sexual e, das 57 mulheres, a maior proporção foi de Regular a Bom (43,86\%), enquanto Nulo a Ruim apresentou a menor proporção (7,01\%). Com o escore total do QS-F, foi possível verificar que $71,93 \%$ das participantes o apresentou adequado, por terem pontuação $\geq 62$ pontos. Concluiu-se que as mulheres avaliadas apresentaram função sexual adequada e satisfatória.

Palavras-chave: climatério, menopausa, sexualidade, comportamento sexual, saúde sexual.

\section{EVALUATION OF SEXUAL FUNCTION OF MENOPAUSAL WOMEN}

\section{ABSTRACT}

The climacteric can present hormonal, physiological and metabolic variation, along with psychosocial changes, that have repercussions on life and sexual interest. The objective was to assess and classify the sexual function of women in menopause. It was recruited 84 women for the research, of which 57 come within inclusion criteria: experiencing menopause (cessation of menstrual cycles for at least 12 months) and having had an active sexual life in the last six months. The Female Sexual Quotient (FSQ) domains were evaluated: desire and sexual interest, foreplay, arousing of the woman and sexual interaction with partner, comfort in sexual intercourse, orgasm and sexual satisfaction. The total score for FSQ determined the performance/sexual satisfaction pattern and, of the 57 women, the major proportion was from regular to good $(43.86 \%)$, while null to bad $(7.01 \%)$ had lowest proportion. The total FSQ score, it was possible to verify that $71.93 \%$ of the participants presented an adequate score, having scored $\geq 62$ points. It was concluded that the evaluated women presented adequate and satisfactory sexual function.

Keywords: climacteric, menopause, sexuality, sexual behavior, sexual health.

\section{INTRODUÇÃO}

A sexualidade é aceita como um dos pilares da qualidade de vida, sendo que o processo de saúde sexual engloba além do bem- estar geral, a identidade sexual estabilizada, função sexual normal e relações sexuais satisfatórias ${ }^{1,2}$. Segundo Kaplan $^{3}$, a resposta sexual pode ser dividida em três fases: desejo, 
excitação e orgasmo. $O$ desejo tem origem no cérebro, já a excitação e o orgasmo envolvem reflexos e estimulações dos órgãos genitais e, cada fase pode apresentar problemas decorrentes de inibidores emocionais e a interrupção de uma ou mais respostas origina as disfunções sexuais.

O desinteresse sexual pode ser apenas uma insatisfação passageira ou uma aversão patológica ao sexo, também chamada de disfunção sexual ${ }^{3-5}$. A disfunção sexual é definida pela Organização Mundial da Saúde (OMS) como a incapacidade de participar do ato sexual com satisfação e, para que uma alteração na normalidade do apetite sexual seja considerada disfunção, a mesma deve causar sofrimento inter e intrapessoal e ser persistente ou recorrente ${ }^{1,6,7}$.

As dificuldades de desempenho e satisfação sexual da mulher se constituem em um relevante problema de saúde, por influenciar negativamente suas experiências sexuais e serem destrutivas perante as sensações de prazer ${ }^{3,5,8,9}$. A causa mais comum para a diminuição da atividade sexual em mulheres com idade mais avançada é a dispareunia, descrita como dor ou desconforto que se inicia com a penetração, durante ou após o ato sexual, sendo causada pela contração involuntária da musculatura vaginal, pela falta de lubrificação da vagina, por infecções urinárias, lesões na vulva ou vagina, entre outros $^{1,2,5,10}$. No entanto, a disfunção sexual também pode estar envolvida com experiências insatisfatórias nas relações sexuais ${ }^{11}$.

Abdo $^{5,8,12}$ afirma que a disfunção sexual é caracterizada pela falta, excesso, desconforto e/ou dor durante o ato sexual, o que interfere em uma ou mais fases da resposta sexual (desejo, excitação e/ou orgasmo), podendo se manifestar de forma persistente e acarretar prejuízos mais complexos.

O climatério de acordo com a OMS tem como definição ser uma fase da evolução biológica da vida da mulher e não patológica, que corresponde a um período de transição do ciclo reprodutivo para não reprodutivo, marcada pela menopausa. Esse período é caracterizado por várias alterações hormonais, fisiológicas e metabólicas que podem vir associadas às mudanças psicossociais ${ }^{13,14}$.

A menopausa é um acontecimento natural da vida da mulher, associado a eventos psicossociais e ao envelhecimento, por isso apresenta necessidade de orientações, de acordo com as preferências e expectativas da mesma.
Essa fase marca o início da vida não reprodutiva da mulher, sendo considerada instalada quando não apresenta menstruações por um período mínimo de 12 meses $^{13,14}$. Nessa etapa ocorrem mudanças hormonais, estéticas, sociais e emocionais, com repercussão direta na vida e no interesse sexual, inibindo ou aumentando anormalmente $o$ apetite sexual, no entanto estudos demonstram que a qualidade da vida sexual dessas mulheres pode continuar satisfatória ${ }^{2,3}$.

Segundo os dados apresentados, essas mulheres irão passar um terço da vida na fase de diminuição do estrogênio, também chamado hipoestrogenismo, que promove sintomas vasomotores, atrofia vaginal, sintomas urinários, aumento de risco para doença cardiovascular e osteoporose, além das disfunções no âmbito sexual ${ }^{13,14}$. Vale destacar que os fatores psicológicos, fisiológicos, e/ou culturais podem influenciar o desejo sexual, inibindo ou aumentando anormalmente 0 apetite sexual dessas mulheres e, por isso, são considerados fatores de risco para o comportamento da sexualidade humana ${ }^{2,3}$.

Ainda assim, as mudanças biológicas do envelhecimento não são os maiores responsáveis pela extinção do comportamento sexual das mulheres após a menopausa. Pode-se considerar também que essa geração de mulheres ainda vive subjugada a uma cultura cheia de tabus e proibições, que as tornaram limitadas e reprimidas sexualmente. Sendo assim, a sociedade é levada a crer que sua sexualidade é sufocada com o avanço da idade, até ser completamente ignorada ${ }^{4,10,16}$.

No entanto, Silveira et al. ${ }^{17}$ demonstram que a terceira idade tende a permanecer ativa sexualmente com o auxílio de medicamentos estimulantes e a sexualidade dessa população que antes era negligenciada, agora é motivo de preocupação devido aos riscos de doenças sexualmente transmissíveis (DST).

De acordo com o Instituto Brasileiro de Geografia e Estatística (IBGE) ${ }^{18,19}$, em 2011 havia 37,7 milhões de mulheres com idade acima de 40 anos, e, em 2016, a expectativa de vida das mulheres passou para 79,4 anos, sendo que, em 1940 era de 48,3 anos. Logo, vários países, inclusive o Brasil, estão tendo sua estrutura etária modificada nos últimos 40 anos, o que é demonstrado pela diminuição na proporção de crianças e jovens simultaneamente a um aumento na população de adultos e idosos na 
conjuntura populacional. Ademais, atualmente, a parcela idosa da população é mais expressiva em quantia absoluta e consideravelmente mais importante em participação social do que há algumas décadas. Essas mudanças vêm acompanhadas de novas demandas relacionadas às políticas públicas de saúde e da necessidade da inserção ativa dos idosos na vida social ${ }^{20}$.

Desse modo, o entendimento sobre a sexualidade na terceira idade é essencial para melhorar a qualidade de vida no processo de envelhecimento da mulher ${ }^{2}$, além de possibilitar estudar intervenções e/ou orientações que melhorem a qualidade de vida sexual dessas mulheres, de modo que o envelhecimento seja acompanhado de uma maior satisfação e qualidade de vida sexual. Sendo assim, o presente estudo teve como objetivo avaliar e classificar a função sexual de mulheres na menopausa.

\section{METODOLOGIA}

Estudo transversal do qual participaram e foram entrevistadas, inicialmente, 84 mulheres, frequentadoras de Unidade Básica de Saúde (UBS) e de um centro de atendimento em Fisioterapia e reabilitação da FCT/UNESP, na cidade de Presidente Prudente. Das entrevistadas, 57 se enquadraram nos critérios estabelecidos: mulheres consideradas sexualmente ativas que apresentaram sua última relação, no máximo, há seis meses, como indicado por $\mathrm{Abdo}^{8,12}$ e $\mathrm{Cabral}^{9}$ e consideradas como mulheres na menopausa, cujas menstruações cessaram por um período de 12 meses ou mais ${ }^{14}$.

Após concordarem com os objetivos e procedimentos do estudo assinaram ao Termo de Consentimento Livre e Esclarecido. O estudo foi aprovado no Comitê de Ética em Pesquisa de Seres Humanos da Faculdade de Ciências e Tecnologia da Universidade Estadual Paulista (FCT/UNESP) com protocolo CAAE no 82745818.4.0000.5402.

Foi aplicado o questionário Quociente Sexual - Versão Feminina (QS-F), o qual é um instrumento de avaliação da função sexual de mulheres, que permite identificar anormalidades, de modo a auxiliar no diagnóstico de Disfunção Sexual Feminina. O QS-F é um questionário de fácil manuseio composto 10 questões auto responsivas, que aborda cinco domínios específicos: Desejo e interesse sexual (perguntas 1, 2 e 8), Preliminares (pergunta 3), Excitação pessoal e sintonia com o parceiro (perguntas $4 \mathrm{e}$ 5), Conforto (perguntas 6 e 7) e Orgasmo e satisfação (perguntas 9 e 10). Para cada questão é ofertada uma escala crescente de 0 a 5 , onde 0 significa "NUNCA" e 5 significa "SEMPRE". O resultado obtido deve ser multiplicado por 2, totalizando em uma soma de 0 a 100 pontos, sendo 0 a condição menos favorável e 100 a condição mais favorável em relação à prática sexual. $O$ escore final deve ser calculado seguindo a fórmula abaixo: Escore final $=2 \times\left(Q_{1}+Q_{2}+Q_{3}+\right.$ $\left.\mathrm{Q}_{4}+\mathrm{Q}_{5}+\mathrm{Q}_{6}+\left[5-\mathrm{Q}_{7}\right]+\mathrm{Q}_{8}+\mathrm{Q}_{9}+\mathrm{Q}_{10}\right)^{8,12}$. Primeiramente, soma-se os escores das questões, incluindo $Q_{7} R$, que é 5 menos o escore da questão 7. E, por fim, multiplica-se o resultado das somas por 2 .

O desempenho sexual geral foi classificado como: Nulo a Ruim (0 a 20 pontos), Ruim a Desfavorável (22 a 40 pontos), Desfavorável a Regular (42 a 60 pontos), Regular a Bom (62 a 80 pontos) ou Bom a Excelente (82 a 100 pontos). Foram consideradas satisfeitas sexualmente, as mulheres que obtiveram escores igual ou maior que 62 pontos, tendo seu padrão de desempenho sexual classificado de Regular a Bom ou de Bom a Excelente $e$ as mulheres que obtiveram pontuação abaixo de 62 pontos foram consideradas sexualmente insatisfeitas ${ }^{9}$.

\section{RESULTADOS}

Foram recrutadas 84 mulheres para a pesquisa, das quais 57 se enquadraram nos critérios da pesquisa e, portanto, foram incluídas no estudo. Destas, 47 participantes $(82,46 \%)$ frequentavam grupos de exercícios em uma Unidade Básica de Saúde (UBS) e 10 (17,54\%) eram atendidas em um centro de atendimento em Fisioterapia e reabilitação da FCT/UNESP, ambos em Presidente Prudente.

A faixa etária das participantes variou de 42 a 69 anos, com uma média de 55,96 $\pm 5,76$ anos. Quando perguntadas sobre a última prática sexual, o período variou de 1 a 30 dias, com média de 8,4 dias desde a última relação.

Sobre o tempo de menopausa, declararam que estavam na menopausa: de 1 a 5 anos 20 participantes (35,09\%), de 6 a 10 anos 15 $(26,32 \%)$, de 11 a 15 anos $8(14,03 \%)$, de 16 a 20 anos $5(8,77 \%)$, há mais de 21 anos $1(1,76 \%)$ e 8 $(14,03 \%)$ declararam ter passado por histerectomia, que é o procedimento pelo qual se faz a ressecção do útero, podendo retirar apenas a parte superior do útero, deixando o colo intacto 
(parcial) ou incluindo a remoção do colo do útero (total) $^{21}$.

E quanto o questionário aplicado, as participantes foram classificadas em nulo a ruim $(7,01 \%)$, ruim a desfavorável $(10,53 \%)$, desfavorável a regular $(10,53 \%)$, regular a bom $(43,86 \%)$ e bom a excelente $(28,07 \%)$. Com relação ao escore de desempenho/satisfação sexual, eram consideradas satisfeitas sexualmente quando a pontuação do QS-F era maior ou igual a 62 pontos e verificou-se que a maior parte das mulheres 41 (71,93\%) alcançaram ou ultrapassa essa pontuação (Tabela

1).

Tabela 2. Porcentagem da pontuação referente ao domínio Desejo e interesse sexual.

\section{Questões \\ Respostas}

1. Você costuma pensar espontânea-mente em sexo, lembra de sexo ou se imagina fazendo sexo?

2. O seu interesse por sexo é suficiente para você participar da relação com vontade?

8. Você consegue se envolver, sem se distrair (sem perder a concentração), durante a relação sexual?
Tabela 1. Pontuação das participantes de acordo com a nota de corte do QS-F.

\begin{tabular}{lcccc}
\hline Corte QS-F & \multicolumn{2}{c}{$<\mathbf{6 2}$ pontos } & \multicolumn{2}{c}{$\geq \mathbf{6 2}$ pontos } \\
\hline Participantes & $\mathrm{N}$ & $\%$ & $\mathrm{~N}$ & $\%$ \\
\hline $57(100 \%)$ & 16 & 28,07 & 41 & 71,93 \\
\hline
\end{tabular}

Com relação aos domínios específicos do QS-F, no domínio Desejo e interesse sexual (perguntas 1,2 e 8 ) verificou-se que, $28(49,2 \%)$ às vezes pensavam espontaneamente em sexo, para $16(28,07 \%)$, na maioria das vezes, o interesse por sexo era suficiente para participar da relação com vontade e, 24 (42,1\%) sempre conseguiam se envolver sem distração durante a relação (Tabela 2).

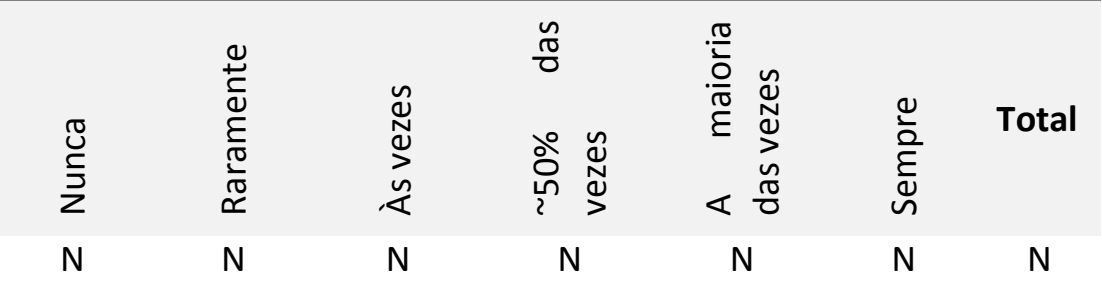

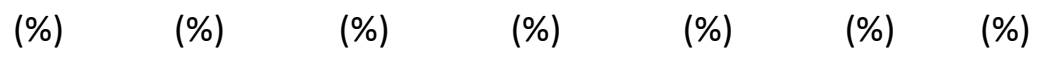

$\begin{array}{ccccccc}5 & 10 & 28 & 2 & 7 & 5 & 57 \\ (8,77) & (17,55) & (49,12) & (3,51) & (12,28) & (8,77) & (100)\end{array}$

$\begin{array}{ccccccc}2 & 7 & 14 & 4 & 14 & 16 & 57 \\ (3,51) & (12,28) & (24,56) & (7,02) & (24,56) & (28,07) & (100)\end{array}$

$\begin{array}{ccccccc}3 & 6 & 7 & 5 & 12 & 24 & 57 \\ (5,26) & (10,53) & (12,28) & (8,77) & (21,06) & (42,1) & (100)\end{array}$

Quanto ao domínio Preliminares (pergunta 3), foi possível constatar que 31 (54,38\%) participantes sempre se sentiam estimuladas pelas preliminares. Ou seja, a maioria das mulheres afirmou que os beijos, carícias e afagos as estimulavam a continuar a relação sexual (Tabela 3). 
Tabela 3. Porcentagem da pontuação referente ao domínio Preliminares.

\begin{tabular}{|c|c|c|c|c|c|c|c|}
\hline \multirow{3}{*}{ Questão } & \multirow{2}{*}{\multicolumn{2}{|c|}{ 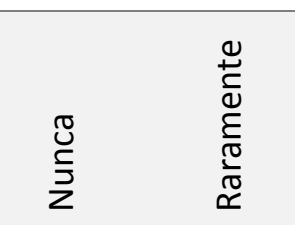 }} & \multirow[b]{2}{*}{ 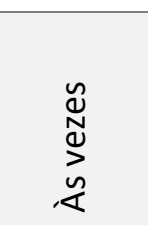 } & \multirow[b]{2}{*}{ 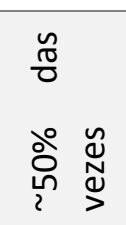 } & \multirow[b]{2}{*}{ 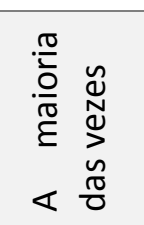 } & \multirow[b]{2}{*}{ 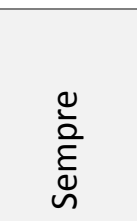 } & \multirow[b]{2}{*}{ Total } \\
\hline & & & & & & & \\
\hline & $\begin{array}{c}N \\
(\%)\end{array}$ & $\begin{array}{c}N \\
(\%)\end{array}$ & $\begin{array}{c}N \\
(\%)\end{array}$ & $\begin{array}{c}N \\
(\%)\end{array}$ & $\begin{array}{c}N \\
(\%)\end{array}$ & $\begin{array}{c}N \\
(\%)\end{array}$ & $\begin{array}{c}N \\
(\%)\end{array}$ \\
\hline $\begin{array}{l}\text { 3. As preliminares (carícias, beijos, } \\
\text { afagos etc) a estimulam a } \\
\text { continuar a relação sexual? }\end{array}$ & $\begin{array}{c}4 \\
(7,02)\end{array}$ & $\begin{array}{c}5 \\
(8,77)\end{array}$ & $\begin{array}{c}9 \\
(15,79)\end{array}$ & $\begin{array}{c}2 \\
(3,51)\end{array}$ & $\begin{array}{c}6 \\
(10,53)\end{array}$ & $\begin{array}{c}31 \\
(54,38)\end{array}$ & $\begin{array}{c}57 \\
(100)\end{array}$ \\
\hline
\end{tabular}

No domínio Excitação pessoal e sintonia com o parceiro (perguntas 4 e 5), 21 (36,84\%) afirmaram que sempre costumavam ficar lubrificadas durante a relação e, 24 (42,1\%) disseram que à medida que a excitação do parceiro aumentava, elas sempre se sentiam mais estimulada para o sexo (Tabela 4).

Tabela 4. Porcentagem da pontuação referente ao domínio Excitação pessoal e sintonia com o parceiro.

Questões

\section{Respostas}

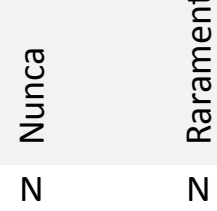

(\%)

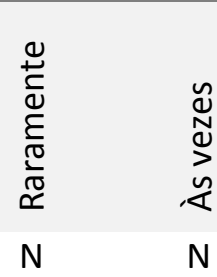

(\%)

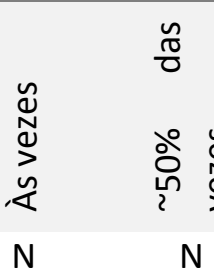

(\%)

(\%)

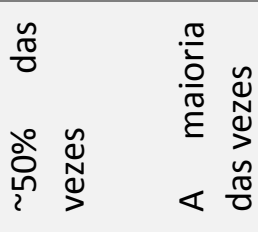

$\mathrm{N}$

(\%)

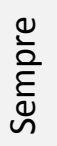

$\mathrm{N}$

(\%)
Total $\mathrm{N}$

(\%)

4. Você costuma ficar lubrificada (molhada) durante a relação sexual?

$\begin{array}{ccccccc}5 & 8 & 10 & 4 & 9 & 21 & 57 \\ (8,77) & (14,03) & (17,55) & (7,02) & (15,79) & (36,84) & (100)\end{array}$

5. Durante a relação sexual à medida que a excitação do seu parceiro vai aumentando, você também se sente mais estimulada

\section{4}

$(7,02)$

5

$(8,77)$

\section{8} $(14,03)$

6

$(10,53)$

\section{0}

$(17,55)$
24

$(42,1)$
57

(100) para o sexo?

Nas questões referentes ao domínio Conforto (perguntas 6 e 7), foi demonstrado que 29 (50.88\%) das participantes sempre relaxavam a vagina o suficiente durante a penetração do pênis e, 26 (45.62\%) nunca sentiam dor durante a relação sexual, quando o pênis penetrava em sua vagina, indicando baixa possibilidade de disfunções como a dispareunia nessa concentração de participantes (Tabela 5). 
Tabela 5. Porcentagem da pontuação referente ao domínio Conforto.

\section{Questões \\ Respostas}

(2)

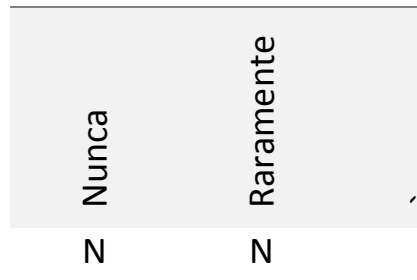

(\%)

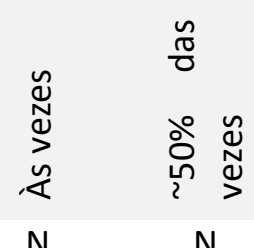

(\%)
(\%)

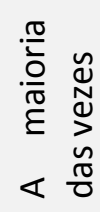

$\mathrm{N}$

(\%)

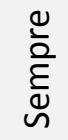

Total

$\mathrm{N}$

(\%)

\section{Durante a relação sexual, você relaxa a vagina suficiente para facilitar a penetração do pênis?}

$\begin{array}{ccccccc}3 & 4 & 8 & 5 & 8 & 29 & 57 \\ (5,26) & (7,02) & (14,04) & (8,77) & (14,03) & (50,88) & (100)\end{array}$

7. Você costuma sentir dor durante a relação sexual, quando o pênis penetra em sua vagina?

$\begin{array}{ccccccc}26 & 8 & 14 & 3 & 1 & 5 & 57 \\ (45,62) & (14,03) & (24,56) & (5,26) & (1,76) & (8,77) & (100)\end{array}$

No que se refere ao domínio Orgasmo e Satisfação (perguntas 9 e 10), foi verificado que 18 (31,58\%) participantes conseguiam, na maioria das vezes, atingir o orgasmo nas relações que realizava e, 15 (26,32\%) sempre conseguiam um grau de satisfação sexual durante a relação que as estimulava a fazer sexo outras vezes, em outros dias.

Tabela 6. Porcentagem da pontuação referente ao domínio Orgasmo e Satisfação.

\section{Questões \\ Respostas}

\begin{tabular}{|c|c|c|c|c|c|c|}
\hline$\frac{\substack{0 \\
5}}{\sum}$ & 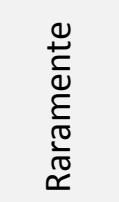 & $\begin{array}{l}\stackrel{n}{N} \\
\stackrel{n}{<}\end{array}$ & 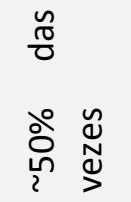 & 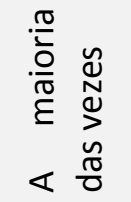 & $\frac{\circlearrowright}{\frac{1}{2}}$ & Total \\
\hline $\begin{array}{c}\mathrm{N} \\
(\%)\end{array}$ & $\begin{array}{c}\mathrm{N} \\
(\%)\end{array}$ & $\begin{array}{l}\mathrm{N} \\
(\%)\end{array}$ & $\begin{array}{l}\mathrm{N} \\
(\%)\end{array}$ & $\begin{array}{l}\mathrm{N} \\
(\%)\end{array}$ & $\begin{array}{c}N \\
(\%)\end{array}$ & $\begin{array}{l}\mathrm{N} \\
(\%)\end{array}$ \\
\hline $\begin{array}{c}4 \\
(7,02)\end{array}$ & $\begin{array}{c}5 \\
(8,77)\end{array}$ & $\begin{array}{c}11 \\
(19,3)\end{array}$ & $\begin{array}{c}6 \\
(10,53)\end{array}$ & $\begin{array}{c}18 \\
(31,58)\end{array}$ & $\begin{array}{c}13 \\
(22,8)\end{array}$ & $\begin{array}{c}57 \\
(100)\end{array}$ \\
\hline $\begin{array}{c}7 \\
(12,28)\end{array}$ & $\begin{array}{c}2 \\
(3,51)\end{array}$ & $\begin{array}{c}14 \\
(24,56)\end{array}$ & $\begin{array}{c}5 \\
(8,77)\end{array}$ & $\begin{array}{c}14 \\
(24,56)\end{array}$ & $\begin{array}{c}15 \\
(26,32)\end{array}$ & $\begin{array}{c}57 \\
(100)\end{array}$ \\
\hline
\end{tabular}

9. Você consegue atingir o orgasmo (prazer máximo) nas relações sexuais que realiza?

10. O grau de satisfação que você consegue com a relação sexual lhe dá vontade de fazer sexo outras vezes, em outros dias?

\section{DISCUSSÃO}

Esta pesquisa investigou a função sexual de mulheres na menopausa usando o questionário QS-F. Tal questionário foi escolhido por permitir avaliar a satisfação e a função sexual, além de possibilitar a distinção de mulheres com e sem disfunção sexual ${ }^{4,8}$. Como principais resultados verificou-se que a maior parte das avaliadas, $71,93 \%$, foram consideradas satisfeitas sexualmente, alcançando ou ultrapassando os 62 pontos pré-estabelecidos e sendo classificadas de Regular à Bom ou Bom à Excelente.

No período da menopausa, o desejo sexual hipoativo é um dos problemas sexuais mais prevalente, seguido pela disfunção da 
excitação e orgasmo em mulheres brasileiras de meia-idade, contradizendo os resultados do presente estudo. Um estudo identificou o desejo sexual hipoativo em aproximadamente $60 \%$ das mulheres nesse período ${ }^{22}$. Estudos conduzidos em outros contextos divulgaram resultados similares, indicando o desejo sexual hipoativo como a alteração sexual feminina mais comum no Egito $(66,4 \%)^{23}$, Índia $(73,2 \%)^{24}$ e Turquia $(60,6 \%)^{25}$.

Existem evidências de que fatores psicossociais, incluindo a qualidade do relacionamento interpessoal, suporte social, bem-estar emocional, doenças crônicas e depressão influenciam a função sexual ${ }^{26}$, assim como a ausência de parceiro ou parceiro com problemas de saúde ${ }^{27}$. No entanto, entre todos os fatores que afetam o desejo sexual feminino, o envelhecimento parece ser o mais significativo ${ }^{28}$. Além disso, as doenças crônicas, que aparecem com o envelhecimento e os tratamentos relacionados, podem afetar direta ou indiretamente a função sexual feminina, pela diminuição dos níveis dos esteroides sexuais, inervação e perfusão sanguínea dos órgãos genitais femininos ${ }^{29}$. Esse fato pode explicar, pelo menos em partes, os resultados do presente estudo, pois a maioria das mulheres se encontrava na meia idade e possivelmente tal aspecto pode ser sido um fator protetor para a ocorrência de DS.

A sexualidade e a prática sexual podem ser prejudicadas pela ocorrência de alterações urogenitais, tais como dispareunia e a diminuição da lubrificação, como resultado do declínio hormonal, fato que não foi observado no presente estudo. Os distúrbios com mais destaque na vida sexual feminina são diminuição da libido, da frequência e da resposta orgástica, cuja ocorrência está relacionada à diminuição do muco cervical e atrofia vulvovaginal, decorrente do hipoestrogenismo ${ }^{30,31}$, além da dor, ardência e desconfortos. Acredita-se que as participantes do presente estudo possam fazer uso da terapia de reposição hormonal, tendo em vista que ela atua sobre aspectos gerais femininos e restabelece a hidratação e lubrificação da mucosa vaginal $\left.\right|^{31}$.

Uma vida sexual ativa e satisfatória durante a menopausa pode ser justificada por fatores como maior companheirismo, presença de novo parceiro sexual, estabilidade financeira, maior tempo dedicado a si mesma, ausência de menstruação e consequente redução das chances de gravidez $^{32,33}$, condição que oferece mais liberdade feminina para expressar sua sexualidade e que autores ${ }^{34}$ destacam que precisa ser entendida considerando-se a vivência, o contexto histórico, social, econômico e cultural em que a mulher se insere.

No entanto, a insatisfação apresentada por $28,07 \%$ das participantes pode estar relacionada à sintomas físicos e/ou emocionais que interferem direta e indiretamente a sexualidade $^{33,34}$. Esse fato norteia para a necessidade de intervenções junto a essa população para que possam alcançar benefícios com a prática sexual. Para tanto, se fazem necessárias, pesquisas mais aprofundadas sobre a atuação fisioterapêutica no tratamento de queixas sexuais de mulheres na menopausa.

Vale destacar que o presente estudo apresenta algumas limitações que devem ser consideradas. Não foi realizada associação com o tempo de menopausa das mulheres avaliadas o que poderia ter enriquecido à discussão do estudo, bem como não foi coletada a informação sobre o possível uso da terapia de reposição hormonal. Por isso, sugerem-se outros estudos epidemiológicos para abordar e correlacionar outros desfechos clínicos que possam se relacionar diretamente à função sexual. No entanto, esse estudo foi importante para a compreensão das condições clínicas e sexuais das mulheres no período da menopausa e com isso proporcionar uma melhor conscientização e orientação sobre sexualidade e função sexual no climatério.

Após a análise dos dados coletados, foi possível verificar que o desempenho sexual da maior parte das mulheres no período da menopausa se apresenta adequado e satisfatório.

\section{CONFLITO DE INTERESSE}

Os autores declaram não haver qualquer potencial conflito de interesse que possa interferir na imparcialidade deste trabalho científico.

\section{REFERÊNCIAS}

1. Lima SMRR, Silva HFS, Postigo $S$, Aoki T. et al. Disfunções sexuais femininas: questionários utilizados para avaliação inicial. Arq Med Hosp Fac Cienc Med Santa Casa São Paulo. 2010;55(1):1-6.

2. De Lorenzi DRS, Saciloto B. Freqüência da atividade sexual em mulheres menopausadas. Rev Assoc Med Bras. 2006;52(4):256-60. DOI: 
http://dx.doi.org/10.1590/S0104-

\section{7}

3. Kaplan HS. Hypoactive sexual desire. J Sex Marital Ther. 1977;3(1):3-9. DOI: https://doi.org/10.1080/00926237708405343

4. Polier AA, Alves TMB. Perfil da satisfação e função sexual de mulheres idosas. Fisioter Mov Curitiba. 2009;22(2):151-8.

5. Abdo CHN, Fleury HJ. Aspectos diagnósticos e terapêuticos das disfunções sexuais femininas. Rev Psiquiatr. 2006;33(3):162-7. DOI: http://dx.doi.org/10.1590/S0101$\underline{60832006000300006}$

6. DataSUS. CID-10 - F52 Disfunção sexual, não causada por transtorno ou doença orgânica. Organização Mundial da Saúde (OMS). Acesso em 2017 Out 20. Disponível em: http://www.datasus.gov.br/cid10/V2008/WebHe Ip/f50_f59.htm\#F52

7. Carvalheira AA, Allen-Gomes F. A disfunção sexual na mulher. In: Oliveira CF (Ed.). Manual de ginecologia. Lisboa: Sociedade Portuguesa de Ginecologia e Obstetrícia. 2011. p. 119-34.

8. Abdo CHN. Elaboração e validação do quociente sexual - versão feminina: uma escala para avaliar a função sexual da mulher. Rev Bras Med. 2006;63(9):477-82.

9. Cabral GO, Almeida MSCTF. Avaliação da qualidade de vida e satisfação sexual em mulheres com incontinência urinária sob tratamento fisioterapêutico. [Dissertação]. Brasília: Centro Universitário de Brasília. 2010.

10. Prado DS, Mota VPLP, Lima TIA. Prevalência de disfunção sexual em dois grupos de mulheres de diferentes níveis socioeconômicos. Rev Bras Ginecol Obstet. 2010;32(3):139-43. DOI: http://dx.doi.org/10.1590/\$0100-

\section{$\underline{72032010000300007}$}

11. Da Fonseca MFSM, Bresin R. Avaliação da função sexual de estudantes de graduação em Enfermagem. Mundo da Saúde. 2008;32(4):4306.DOI:

https://doi.org/10.15343/0104-
12. Abdo CHN. Quociente sexual feminino: um questionário brasileiro para avaliar a atividade sexual da mulher. Diagn Tratamento. 2009;14(2):89-91.

13. Peres SMV, Pinto MJC. Qualidade da função sexual feminina na pós-menopausa. Rev Latinoam Med Sexual. 2012;1(2):12-9.

14. Navega MT, Oishi J. Comparação da qualidade de vida relacionada à saúde entre mulheres na pós-menopausa praticantes de atividade física com e sem osteoporose. Rev Bras de Reumatol. 2007;47(4):258-64. DOI: http://dx.doi.org/10.1590/S0482$\underline{50042007000400004}$

15. Hoga L, Rodolpho J, Gonçalves B, Quirino B. Women's experience of menopause: a systematic review of qualitative evidence.JBI Database Systematic Rev Implementation Rep. 2015;13(8):250-337. DOI: http://dx.doi.org/10.11124/jbisrir-2015-1948

16. Costa VDM, Pinto MJC. Análise prospectiva da resposta sexual feminina na gestação. Rev Latinoam Med Sexual. 2012;1(1):5-13.

17. Silveira MM, Batista JS, Colussi EL, Wibelinger LM. Sexualidade e Envelhecimento: discussões sobre a AIDS. Rev Temática Kairós Gerontol. 2011;14(5):205-20.

18. Cintra SV, Fabiano MD, Carvalho PB. Políticas públicas para mulheres: uma análise do município de Ituiutaba (MG). Rev Conexões Gerais. 2015;3:1-86.

19. Agência de Notícias IBGE. Em 2016, expectativa de vida era de 75,8 anos. Acesso em 19 mai 2019. Disponível em: https://agenciadenoticias.ibge.gov.br/agenciasala-de-imprensa/2013-agencia-denoticias/releases/18470-em-2016-expectativade-vida-era-de-75-8-anos

20. Closs VE, Schwanke CHA. A evolução do índice de envelhecimento no Brasil, nas suas regiões e unidades federativas no período de 1970 a 2010. Rev Bras Geriatr Gerontol. 2012;15(3):443-58. DOI: $\quad$ http://dx.doi.org/10.1590/S1809$\underline{98232012000300006}$ 
21. Piazza MJ, Peixoto AP, Peixoto RN, Urbanetz AA. Histerectomia total versus histerectomia supracervical. Femina. 2011;39(10):479-84.

22. Valadares ALR, Pinto-Neto AM, de Souza MH, Osis MJD, Paiva LHSC. The prevalence of the components of low sexual function and associated factors in middle-aged women. J Sex Med. 2011;8(10):2851-8. DOI:

http://dx.doi.org/10.1111/j.1743$\underline{6109.2011 .02405 . x}$

23. Hassanin IMA, Helmy YA, Fathalla MMF, Shahin AY. Prevalence and characteristics of female sexual dysfunction in a sample of women from Upper Egypt. Int J Gynaecol Obstet. 2010;108(3):219-23. DOI: http://dx.doi.org/10.1016/j.ijgo.2009.09.031

24. Singh JC, Tharyan P, Kekre NS, Singh G, Gopalakrishnan G. Prevalence and risk factors for female sexual dysfunction in women attending a medical clinic in south India. J Postgrad Med. 2009;55(2):113-20.

DOI:

http://dx.doi.org/10.4103/0022-3859.52842

25. Aslan E, Beji NK, Gungor I, Kadioglu A, Dikencik BK. Prevalence and risk factors for low sexual function in women: a study of 1,009 women in an outpatient clinic of a university hospital in Istanbul. J Sex Med. 2008;5(9):204452. DOI: http://dx.doi.org/10.1111/i.1743$\underline{6109.2008 .00873 . x}$

26. Abdo CHN, Valadares ALR, Oliveira Jr WMO, Scanavino MT, Afif-Abdo J. Hypoactive sexual desire disorder in a population-based study of Brazilian women: associated factors classified according to their importance. Menopause. 2010;17(6):1114-21.

DOI: http://dx.doi.org/10.1097/gme.0b013e3181e197 $\underline{55}$

27. Gott $M$, Hinchliff $S$. How important is sex in later life? The views of older people. Soc Sci Med. 2003;56(8):1617-28.

DOI: https://doi.org/10.1016/S0277-9536(02)00180-6

28. Hayes RD, Dennerstein L, Bennett CM, Koochaki PE, Leiblum SR, Graziottin A. Relationship between hypoactive sexual desire disorder and aging. Fertil Steril. 2007;87(1):10712. DOI:
29. Basson R, Schultz WW. Sexual sequelae of general medical disorders. Lancet. 2007;369(9559):409-24. DOI: https://doi.org/10.1016/S0140-6736(07)60197-4

30. Alves ERP, Costa AM, Bezerra SMMS, Nakano AMS, Cavalcanti AMTS, Dias MD. Climatério: a intensidade dos sintomas e o desempenho sexual. Texto Contexto Enferm. 2015;24(1):64-71. DOI: $\quad$ http://dx.doi.org/10.1590/0104$\underline{07072015000590014}$

31. Nappi RE, Kingsberg S, Maamari R, Simon J. The CLOSER (Clarifying vaginal atrophy's impact on sex and reationships) survey: implications of vaginal discomfort in postmenopausal women and in male partners. J Sex Med. 2013;10(9):2232-41.

DOI:

http://dx.doi.org/10.1111/jsm.12235

32. Falcke D, Zordan E. Amor, casamento e sexo: opinião de adultos jovens. Arq Bras Psicol. 2010;62(2):143-55. DOI:

http://dx.doi.org/10.1590/S1414$\underline{98932002000200009}$

33. Ashdown BK, Hackathorn J, Clark EM. In and out of the bedroom: sexual satisfaction in the marital relationship. J Integrated Soc Sci. 2011;2(1):40-57.

34. Araújo IA, Queiroz ABA, Moura MAV, Penna LHG. Social representations of the sexual life of climacteric women assisted at public health services. Texto Contexto Enferm. 2013;22(1):11422. DOI: http://dx.doi.org/10.1590/S0104$\underline{07072013000100014}$

\section{https://doi.org/10.1016/i.fertnstert.2006.05.071}

